\title{
NEWTON AND THE SCIENCE OF HIS AGE
}

\author{
By Prof. E. N. DA C. ANDRADE, F.R.S.
}

$\mathbf{I}_{\mathrm{t}}^{\mathrm{T}}$ $T$ is my task-my honourable and inspiring taskto say something of Isaac Newton as seen against the background of the science of his time. I shall try to display briefly the position as he found it and to resume in a small space his great achievements and the changes in outlook which they produced. In praising Newton I shall endeavour to do justice to his great forerunners and to the men of his time who pursued worthily the same great ends as he did, and who would have held the centre of the stage in any other age than that dominated by him. For Newton, like Shakespeare, did not stand as a lonely adventurer into new realms, though he travelled farther and straighter than the rest. Shakespeare was the supreme poet and playwright at a time when poetry and plays were part of the life of every cultivated man and occupied the attention of the brightest intellects. Newton was the supreme scientist in an age when the quantitative method of questioning Nature was abroad in the air. Each was the child of his time.

Let us consider the position when Newton went to Cambridge in 1661. The hold of Aristotle, whose works had for centuries been the ultimate resort of all those seeking knowledge of the working of Nature, had been shaken off by such men as Galileo and Gilbert, but most of the learned still thought that those who relied on experiment were pursuing a futile and impudent course. The first resolve of Marlowe's Faustus

"Having commene'd, be a divine in shew,

Yet level at the end of every art,

And live and die in Aristotle's works"

still represented the aim of many students. The foundation of the Royal Society in 1662 had been the occasion of many attacks on the experimental method, attacks stoutly met by Glanvill and Sprat, and as late as 1692 Sir William Temple's "Essay upon the Anoient and Modern Learning", satirized by Swift in "The Battle of the Books", set out to prove the superiority of the philosophers of the ancient world over all the moderns. Thus when Newton was a young man the new experimental method of questioning Nature was steadily making its way and the omniscience of the ancients was being called in doubt by a new school, but experimental science was by no means firmly established as a respectable study.

The great figures among the worthies of the exact sciences who had already appeared at that time were Copernicus, Tycho Brahe, Kepler, Gilbert, Galileo and Descartes. Kepler, following his great forerunners, had found the true laws of planetary motion, which were to be explained by Newton. Kepler's views as to the mechanism of the planetary motions were in his earlier writings largely mystical, involving the perfect properties of the five regular solids and also certain motivating souls or spirits. Throughout he held to the medieval point of view that a body could not maintain its motion unless there were a force propelling it. In his later writing he invoked a magnetic foree, but it was not directed to the sun, like the true gravitational force, but pushed the planets on their way-"non est attractoria sed promotoria". It was essentially bound up with the rotation of the sun. Thus he was ignorant of the basio laws of mechanics, and his magnetic force had none of the true properties of magnetic forces. Kepler made no approach to a mechanical explanation of his laws. Gilbert had not only established the basic principles of terrestrial magnetism and carried out fundamental work on electricity, but also had invoked a force from the moon-a magnetic force it is trueto produce the tides. Galileo's greatest achievement had been to lay the foundations of mechanies.

None of these men, however, had made any impression on the bulk of the learned: Francis Bacon, for example, does not mention Galileo or Kepler, and refuses to take Gilbert seriously. The great figure in the eye of natural philosophers was Descartes, who had developed a cosmogony based upon mechanical principles, not precise mechanical principles it is true, but principles very different from the mystical ones then in vogue. Joseph Glanvill used to lament that his friends had not sent him to Cambridge, where he might have learned the new philosophy of Descartes, rather than to Oxford, where Aristotelianism ruled. Descartes' system had acquired such a hold on men's minds that his views were still supported long after Newton's death. Both Jean Bernoulli, who died in 1748, and Fontenelle, who died in 1757, were Cartesians to the end. The demolition of the Cartesian system was, in contemporary eyes, one of Newton's greatest achievements. James Thomson said, in his "Ode to the Memory of Sir Isaac Newton", published immediately after his death :

"The heavens are all his own; from the wild rule

Of whirling vortices, and circling spheres,

To their first great simplicity restored.

The schools astonished stood."

From the "Principia" it is clear that Newton himself derived particular satisfaction from having invalidated the Cartesian system. It is fitting, then, that we start our consideration of contemporary science by a glance at this system, not only because it was the only attempt before Newton to explain the motions of heavenly bodies on general principles, but also because it furnishes a contrast which brings out the essential Newtonian point of view.

Descartes starts, in the spirit of medieval thinkers, from certain general philosophical principles. He decides that the fundamental property of matter is extension-impenetrability, colour, hardness and so on are only secondary characteristics. Extension, which has three directions, is the subject of mathematics: motion is the subject of mechanics. All the different qualities of different kinds of matter are provided by different motions of the minute parts of which they are composed. "Give me extension and motion," declares Descartes, "and I will construct the world." One consequence of his fundamental belief is that there cannot be a vacuum, for extension without matter is a contradiction. $\mathrm{He}$ further considers, on theological grounds, that the quantity of motion in the heavens must be constent. He blames Galileo for founding his mechanies on experiments and not on reflexions on first causes. "Every- 
thing Galileo says about the philosophy of bodies falling in empty space is built without foundation: he ought first to have determined the nature of weight." Newton's point of view, of course, was the exact opposite to that of Descartes : he says in the famous letters to Bentley, ". . . for the cause of gravity is what I do not pretend to know, and therefore would take some time to consider of it"; and again, "gravity must be caused by an agent acting constantly according to certain laws, but whether this agent be material or immaterial, I have left to the consideration of my readers". For Newton, as for the best of his successors, science was concerned with the question of "How ?": Descartes, like the ancients, was concerned with the insoluble question of a fundamental "Why ?".

It followed from the philosophic hypothesis of Descartes that the only kind of motion possible in a plenum was a motion in closed paths, more particularly a circular motion, since a particle could only move if another particle took its place. It was on grounds of this kind that he elaborated his vortex hypothesis. Certain very fine particles, which filled interplanetary space, moved round ceaselessly in huge vortices and carried the planets with them. The moon was carried round the earth by a minor vortex, and so on.

Descartes' cosmogony, then, was founded on a philosophical system : it was pictorial and unquantitative. The paths of the comets handed on from one vortex to another were in particular irreconcilable with observation. There was no attempt to deduce Kepler's laws, or to show how anything but circular motion could result from the vortices. The whole scheme was spun from the brain of Descartes, with more or less casual references to actual phenomena. No doubt it was referring to Descartes that Roger Cotes said in his preface to the second edition of the "Principia", "Those who fetch from hypothesis the foundation on which they build their speculations may form indeed an ingenious romance, but a romance it will still be". In spite of, or perhaps because of, this, Descartes' influence was immense, and when Newton was at Cambridge as a young man it was Descartes who was the great authority for all such as speculated on the structure of the universe.

We now turn to the astonishing story of the birth of the "Principia". The time was ripe for the appearance of this great work. As regards the laws of motion, Galileo, whose services Newton freely acknowledges, and Descartes himself had done much to prepare the way for the more precise and particular formulation which Newton gives. Hooke among others had clearly expressed the protest of the most forward spirits of the time against the speculative method. "The truth is, the Science of Nature has been already too long made only a work of the Brain and the Fancy : it is now high time that it should return to the plainness and soundness of Observations on material and obvious things." The Royal Society was active in stimulating the pursuit of the new method, and on the Continent the Accademia del Cimento had done excellent work, while the Académie des Scienees was founded in 1666. These Continental societies had little influence on Newton, but are symptomatic of the general movement towards the experimental method. The stage was set for great things.

The story opens at Woolsthorpe, Newton's birthplace, whither Newton had come from Cambridge in June 1665 to escape the plague. He was then twenty-two years old and not yet a master of arts or fellow of Trinity. He had read what he calls "Schooten's Miscellanies" (probably the "Exercitationum Mathematicarum Libri V"), Descartes' "Geometry" and Wallis' works, and further was, of course, familiar with the work of his teacher Barrow. He had written his first treatise on the calculus, or 'fluxions' as he called it, but he had published nothing. The words which he wrote some fifty years later about this great springtime of his intellectual life have often been quoted but cannot well be omitted on an occasion like this. "In the same year (1666) I began to think of gravity extending to the orb of the moon, and having found out how to estimate the force with which a globe revolving within a sphere presses the surface of the sphere, from Kepler's Rule of the periodical times of the planets being in a sesquialterate proportion of their distance from the centre of their orbs I deduced that the forces which keep the planets in their orbs must [be] reciprocally as the squares of their distances from the centres about which they revolve : and thereby compared the force requisite to keep the moon in her orb with the force of gravity at the surface of the earth, and found them answer pretty near. All this was in the two plague years of 1665 and 1666 , for in those days $I$ was in the prime of my age for invention, and minded mathematics and philosophy more than at any time since. What Mr. Huygens has published since about centrifugal forces I suppose he had before me."

It seems likely that he had already his laws of motion in his head-in any case it is clear that he was convinced that every body would continue to move uniformly in a straight line unless some force acted on it, and that, therefore, there must be some force acting on the moon which drew it away from the straight line, tangential at any moment to its path, in the direction of the earth. Treating the moon's path as circular, from Kepler's third law, and from the law connecting the centrifugal force with the radius and the velocity, or the equivalent proposition to which Newton refers, it is easy to deduce the inverse square law. To show that the force keeping the moon in her orbit is the earth's gravitational force, assumed to diminish as the inverse square, is, however, a further step demanding a computation of how strong the gravitational force at the moon's orbit will be, compared to the measured force at the surface of the earth. Newton made this step and found it "answer pretty near". Why, then, did he delay the announcement of the law of gravity for twenty years or so?

There is always a ready answer to questions of this kind where Newton is concerned-that he never published anything until invited, in general strongly urged, to do so. It is, however, clear from many signs that Newton was not himself satisfied about the matter until some time about 1685 . The usual story is that he took a wrong radius for the earth, namely one corresponding to 60 miles for $1^{\circ}$ of latitude instead of the correct value of about 70 , but this story is very improbable on many grounds, one of which is that good values were readily available to him.

The real reason for Newton putting the work aside seems to have been that the calculation, so far as the force at the earth's surface is concerned, depends essentially upon it being legitimate to assume that the earth's mass may be considered as concentrated at the centre. That this assumption is valid is far from obvious. It is fairly clear from certain passages in "De Motu" and in the "Principia" that it gave Newton some trouble to prove this assumption and 
that he did it late. In a letter to Halley of June 20, 1686, he says, "I never extended the duplicate proportion lower than to the superficies of the earth, and before a certain demonstration $I$ found the last year, have suspected that it did not reach accurately enough down so low". Although this refers to the gravitational force within a sphere, this and the point under discussion are involved in the same mathematical demonstration, which Newton gives in the "Principia" in Book 1, Proposition LXXI and other propositions following it. In any case Newton appears not to have been satisfied with his first calculations and to have turned to other things, possibly his optical experiments.

It is a strange thing that the "Principia" owes its publication largely to a quarrel with Hooke, and its sequel. In 1679 Hooke, then acting as secretary of the Royal Society, wrote to Newton about various scientific matters and asked him very civilly for a philosophic communication-a paper as we should say nowadays. He also asked for Newton's opinion on his "Potentia Restitutiva" and "particularly if you will let me know your thoughts of that of compounding the celestial motions of the planets of a direct motion by the tangent and an attractive motion towards the central body". In his reply Newton made an extraordinary remark. "But yet my affection to philosophy being worn out, so that I am almost as little concerned about it as one tradesman uses to be about another man's trade or a countryman about learning, I must acknowledge myself averse from spending that time in writing about it which I think I can spend otherwise more to my own content and the good of others: and I hope neither you nor anybody else will blame for this averseness." This is but one of many occasions on which Newton expresses his disinclination, almost distaste, for any further scientific work, his first antipathy having been aroused by the disputes and misunderstandings consequent on the publication of his first great work on the prism.

Nevertheless, Newton did comply with Hooke's request for something for the Society by pointing out that a body let fall from on high should strike the earth slightly east of the perpendicular, and gives precise and excellent directions for following out the experiment. The purpose was to prove the diurnal rotation of the earth. Hooke, in reply, pointed out that the ball should fall to the south as well as to the east, and further corrected Newton in a point which is too complicated to discuss here and one on which differences of statement can be due to different interpretations of the problem. This correction, tactlessly expressed, irritated Newton in the highest degree and he answered curtly. In further letters, written in apparent unconsciousness of the annoyance he had given, Hooke suggested quite explicitly that the law needed to explain the planetary motions was the inverse square law.

Now other men had come to the same conclusion. In particular, Wren and Halley had discussed with Hooke the possibility of explaining the mechanism of the heavens on the basis of an inverse square law. Hooke declared that he could demonstrate mathe. matically that the path of a particle in a central inverse square field would be an ellipse, but it is clear that he never did so, and equally clear that he had not the mathematical equipment necessary to begin an attack on the problem. He was in the unfortunate position of being entirely convinoed of a truth that he could not prove. In August 1684 Halley visited
Cambridge and asked Newton what the path would be. He replied that it would be an ellipse and that he had formerly calculated it. He could not find the calculation but soon sent a proof (or apparently two different proofs) to Halley.

This incident seems to have aroused Newton from the distaste for science into which he had fallen, and he put together the treatise "De Motu", founded on a course of lectures, which Halley presented to the Royal Society on December 10, 1684. The story of how Halley then coaxed and cajoled Newton into writing the "Principia" is familiar, but we in the Royal Society ought never to celebrate the great work without a tribute to Halley, who not only realized at once the fundamental importance and significance of Newton's work but also used all his tact to get the book written and made himself financially responsible for the production, the Royal Society being in financial difficulties at that time. We are not now in financial straits and shall, I believe, be glad to bear the expense of producing a second "Principia" when the genius of our age brings it forth. The book appeared in 1687 , and bears the imprimatur of the then president, who, although he achieved nothing in science, is still remembered. He was Samuel Pepys.

The "Principia" is not an easy book to read. The proofs are all given in the form of classical geometry, although, since it is certain that at the time when it was written Newton was in possession of the fundamental processes of the calculus and of the methods of analytical geometry, it is unlikely that this was the form in which he first derived them. Whewell has said, "Nobody since Newton has been able to use geometrical methods to the same extent for the like purposes; and as we read the 'Principia' we feel as when we are in an ancient armoury where the weapons are of gigantic size; and as we look at them we marvel what manner of man he was who could use as a weapon what we can scarcely lift as a burden". Various conjectures have been made by Rosenberger, Cantor, Giesel, Gerhardt and others as to why he did not use the new methods : Rouse Ball thinks that he was probably unwilling to add to the difficulties by introducing a new mathematical method. Be that as it may, he had a horror of unfounded criticism, and, as he told Dr. Derham, "to avoid being baited by little smatterers in mathematics, he designedly made his 'Principia' abstruse ; but yet so as to be understood by able mathematicians". That he did not underrate the difficulty of the work is clear from what he says in the beginning of Book III : "I chose to reduce the substance of that book into the form of propositions (in the mathematical way) which should be read by those only, who had first made themselves masters of the principles establish'd in the preceding books. Nor would I advise any one to the previous study of every proposition of those books. For they abound with such as might cost too much time, even to readers of good mathematical learning. It is enough if one carefully reads the definitions, the laws of motion, and the first three sections of the first book."

The first book contains eertain definitions of space, mass and time which have afforded the more metaphysically inclined a theme ample enough for diseussion. But we will here let them pass uncommented. The famous laws of motion owe much to the labours of previous workers, in particular to Galileo, whose services Newton clearly acknowledges. The simple laws of central orbits under an inverse square law are worked out in much detail and the laws of 
pendulum motion are developed, with due acknowledgments to Huygens, who had published his "Horologium Oscillatorium" in 1673.

In the first book the motions are all supposed to take place in a non-resistant medium. In the second Newton considers motions in a resisting medium, always with his eye on the Cartesian world system which he was intent to demolish. $\mathrm{He}$ deals with a resistance proportional to the velocity and a resistance proportional to the square of the velocity, and further points out different kinds of fluid resistance, which he later defines most clearly in the 28th Query appended to the third edition of the "Opticks", "for the resisting Power of the fluid Medium arises partly from the Attrition of the Parts of the Medium and partly from the Vis inertiae of the Matter", that is, partly from the viscosity and partly from the bulk motion of the medium. The latter resistance he assumes proportional to the square of the velocity. In this book he opens the way to the hydrodynamics of real fluids. In the part dealing with hydrostatics he proves the law of the diminution of atmospherie pressure with height. He then discusses the motion of the pendulum and is the first to suggest its use for making a survey of the gravitational acceleration. In another connexion he derives an expression for the velocity of sound, this being the first case of a calculation of the velocity of a wave from the properties of the medium. The only other point in this book to which I will refer is the calculation which Newton carries out on the motion of an infinitely extended viscous fluid in which a body rotating upon its axis is immersed. What he has in mind is the Cartesian vortex-" "I have endeavoured in this proposition to investigate the properties of vortices, that I may find whether a celestial phenomenon can be explained by them". He effectively defined the force "arising from the want of lubricity in the parts of the fluid" as proportional to the velocity gradient, whence the term 'Newtonian viscosity'. He is thus the first to touch the mathematics of viscous fluids. He finds that the periodic time of circulation of the fluid carried round by the rotating sphere is proportional to the square of the distance from the centre of the sphere*, which is grossly inconsistent with Kepler's third law. Newton considers that his deduction is a clear refutation of the Cartesian vortices and, for once, almost gloats over his victory. "Let philo. sophers then see how the phenomenon of the sesquialterate ratio can be accounted for by vortices". He brings many other objections against the Cartesian vortices : he points out, for example, that a continuous supply of energy will have to be given to the sphere to maintain the motion, because "it is plain that the motion is proportionally transferred from the centre to the circumference of the vortex, till it is quite swallowed up and lost in the boundless extent of that circumference".

The second book of the "Principia" is the foundation stone of mathematical physics. In it Newton shows an extraordinary instinct for grasping the essentials of a problem-it would almost seem that he knew the solution in advance and added the proof as a concession to those less clear-sighted. The manner of his refutation of the Cartesian vortices introduces a new spirit into the discussion: no hypothesis about the heavens is tenable unless the

- Actually this is an error, which appears never to have been pointed out although Stokes has indicated the like error in the case of the rotating cylinder, which Newton works out. It should be the cube of the distance. quantitative deductions from it agree with observations.

The third book opens with an introduction where, after stating what has been done in the first two books, Newton sets down the superb sentence, "Super. stat ut $\mathrm{ex}$ iisdem principiis doceamus constitutionem systematis mundani" - it remains that from the same principles we demonstrate the form of the system of the world. It is this third book which based celestial mechanics so firmly that what was done in the next two hundred years was rather extension of, and improvements on, the Newtonian method than anything radically new. Not only does Newton establish the movements of the setellites of Jupiter, Saturn and the earth, and of the planets round the sun (or rather, as he points out, round the centre of gravity of the solar system) in terms of his gravitational theory, but also he shows how to find the masses of the sun and planets in terms of the earth's mass, which he estimates quite closely to the present accepted value; he accounts for the flattened shape of the earth and other planets; calculates the general variations of $g$ over the surface of the earth; explains the precession of the equinoxes by consideration of the non-sphericity of the earth; calculates the main irregularities of the motion of the moon and of other satellites from the perturbing effect of the sun; explains the general features of the tides; and finally treats the orbits of comets in a way that showed that they were members of the solar system and enabled the returm of Halley's comet in 1759 to be accurately calculated. This brief and imperfect catalogue is merely a reminder of the scope of this extraordinary book, which drew from Laplace, no enthusiast,". . . all this, presented with much elegance, assures to the 'Principia' preeminence over all the other productions of the human mind". The book closes with the famous general scholium which returns to the confutation of Descartes' vortices and says, concerning the cause of gravity, "Hypotheses non fingo".

Although the book was eagerly bought, the Newtonian method and discoveries made way but slowly. Biot says that of Newton's contemporaries three or four only were capable of understanding the "Principia", that Huygens only half adopted the ideas, Leibniz and Jean Bernoulli fought against them, and that fifty years had to pass before the great truth demonstrated by Newton was understood by the generality of men of science, let alone developed. No doubt the difficulty of the book had much to do with the tardy appreciation, outside a narrow circle, of its contents. The Cartesian scheme was easy, pictorial, general : the Newtonian difficult, mathematical, precise. The very method of attacking the problem was altogether new. The second edition appeared in 1713, edited by Roger Cotes, of whom Newton said, "if Mr. Cotes had lived we might have known something". The preface clearly shows that among the leamed the Newtonian scheme had not been widely accepted. On the Continent the progress was still slower and it is generally held that it was Voltaire's "Elémens de la Philosophie de Neuton", which appeared in 1738, that led to Newton's work being appreciated in France. Incidentally, the story of the apple, which Voltaire had from Newton's niece, Mrs. Conduitt, first appeared in the second edition of Voltaire's book, in 1741. Later, it was in France that Newton's work was raised to great glory, when Lagrange and Laplace erected edifices of splendour and elegance on Newtonian foundations. 
The optical work of Newton was perhaps his favourite study : at any rate it was that to which he gave what is probably the only enthusiastic reference which he ever permitted himself, when he said, of his fundamental discovery in the matter of prismatic colours, "being in my judgment the oddest, if not the most considerable detection which hath hitherto been made in the operations of nature". I do not propose to say anything of the beautiful experiments which form the backbone of the book, but a few words as to the Newtonian attitude to the theoretical aspect may be permitted.

The nature of colour had from the days of Aristotle been the subject of philosophical speculation. According to Aristotle, colours are a mixture of light and darkness, or of white and black, a view which, embellished and modified in various ways, survived Newton and appeared again in, for example, Goethe's writings. Descartes was apparently the first to break really new ground in comparing colours to notes in music : his view of light was that it was a pressure transmitted through the particles that filled all space, and he conjectured that a rotation of the particles might be the effective cause of colour, a view which presents inherent difficulties effectively exposed by Hooke. We may agree with Huygens that "Descartes has said nothing that is not full of difficulties or even inconceivable, in dealing with light and its properties". The medieval question as to whether light was a substance or an accident was still occupying the attention of even acute experimentalists such as Grimaldi, the discoverer of diffraction, whose book appeared in 1665. It was Hooke again, who, with his extraordinary flair for the truth, combined with an inability to overcome the last difficulties that stood between him and a convincing conclusion, initiated the modern views. In his "Micrographia", 1665, he expressed the view that light was a very quick vibration propagated with a finite velocity. He gave what is something like Huygens' construction for finding the wave front on refraction, but, since he thought that light travelled faster in a solid medium, such as glass, than in air, he found that in the medium the wave front must make an acute angle with the ray. It was with this 'obliquity' that he connected colour. "Blue is an impression on the Retina of an oblique and confus'd pulse of light, whose weakest part precedes, and whose strongest follows. . . Red is an impression on the Retina of an oblique and confus'd pulse of light, whose strongest part precedes and whose weakest follows." For him blue and red were the primary colours, all others being mixed. There is no time to follow his extraordinarily acute experiments on the colours of thin plates, but it must be noted that his ill-tempered attacks were the cause of Newton expressing a disgust with science which nearly caused him to abandon her pursuit, and were, it is almost cortain, the reason why the "Opticks" was not published until 1704, the year following Hooke's death. The quarrel between the two men, both, as is evident from their correspondence, capable of generous appreciation of the other's achievements, was exacerbated by Oldenberg, then secretary of the Royal Society, whose dislike of Hooke may have sprung from causes little creditable to him. Newton had been undoubtedly stimulated by his reading of Hooke's "Micrographia", and he was always very remiss in acknowledgments to hirn. Let us pay a tribute to poor Hooke, sickly and without position or power- ful friends. He had not Newton's power of thought, but he was probably the most ingenious contriver who ever lived and was a shrewd and daring speculator.

Nowhere more than in his writings on light does Newton stress his dislike of speculation not firmly rooted in experiment. The first words of the "Opticks" are "My design in this Book is not to explain the Properties of Light by Hypotheses, but to propose and prove them by Reason and Experiments", and again, in the 3lst Query appended to the third edition of that book, he makes his point of view very clear, saying "These Principles I consider not as occult Qualities, supposed to result from the specifick Forms of Things, but as general Laws of Nature, by which the Things themselves are form'd: their Truth appearing to us by Phaenomena, though their Causes be not yet discover'd. For these are manifest Qualities, and their Causes only are occult. And the Aristotelians gave the Name of occult Qualities not to manifest Qualities, but to such Qualities only as they supposed to lie hid in Bodies, and to be the unknown Causes of manifest Effects. . . To tell us that every Species of Things is endow'd with an occult specifick Quality by which it acts and produces manifest Effects, is to tell us nothing." It is in the light of this that we must read the famous "Hypotheses non fingo". Newton, of course, did make hypotheses, and even called them such-for example, in "Principia", Book II, Section IX, his assumption as to the be haviour of viscous fluids is headed "Hypothesis", and there are other instances. All he meant was that he was reluctant to speculate beyond any possibility of quantitative deduction, to form conjectures the defence of which would be merely a matter of dialectics.

Newton's corpuscular hypothesis to account for the experimental behaviour of light is clearly a hypothesis, but we shall see how closely he adapts it to the observations. First, he points out that light cannot be a wave motion, or it would spread out on passing through an opening. He knew, it is true, the phenomena of diffraction, but he did not realize how by making the wave-length small enough this could be reconciled with the general facts of rectilinear propagation, for which a stream of particles seems best suited. $\mathrm{He}$ clearly saw that the phenomena of the colours of thin plates demanded a periodicity, and he introduced this into his theory by the hypothesis of fits of easy reflexion and easy transmission. It is clear that light is partly reflected and partly transmitted at the surface of a transparent body : Newton supposed that a light particle alternated at regular intervals between a state in which it was transmitted through the surface and one in which it was sent back. He put forward diffidently ("Those that are averse to assenting to any new Discoveries but such as they can explain by an Hypothesis, may for the present suppose ....") the idea that the impact of the particle on the surface excited vibrations in the medium which, overtaking the particles, put them into these alternating states. We are forcibly reminded of modern theories. The length of the interval of the fit, corresponding to our wave-length, was greater for the red than for the blue, and Newton gives the interval for yellow light incident normally as just about the actual wave-length of yellow light. Unfortunately, the length of the fit depended on the angle of incidence. However, of his whole theory he says, "But whether this hypothesis be true or false I do not here con- 
sider. I content myself with the bare Discovery that the Rays of Light are by some cause or other alternately disposed to be reflected or refracted for many vicissitudes". When he comes to consider polarization, he has to endow his particles with 'sides', so that there was a lack of complete symmetry about the direction of propagation. In short, he gave his particles just those properties which interpreted the experiments and hence was led to endow them with a periodicity and a polarity. This brought him to assume subsidiary waves accompanying the particles when they interacted with matter.

There seems to be a general belief that Huygens, as against Newton, advocated something very close to the wave theory of light, as it was accepted in, let us say, 1900. Huygens' wavelets, however, lacked the essential properties with which Fresnel afterwards endowed them : they were not only longitudinal but also had what are practically particle properties, as exemplified by the fact that the pole, the place where the wavelet touches the envelope, alone is efficacious. He was just as incapable as Newton of giving a satisfactory explanation of diffraction.

If we are asked to state in a sentence what was the main effect of Newton's work on the thought of his time, I think that the answer must be that it was to establish the power and universality of the methods of quantitative science. To Galileo we owe the great service, one that cannot be too highly praised, of having made particle dynamics into a science, but he did not look beyond the earth for its efficacy or suggest the application of his methods to the relative movement of the parts of a continuous medium, such as water. Huygens founded the study of rigid dynamics. Hooke suggested and speculated with extraordinary ingenuity and acuteness. Newton, however, showed that three clearly enunciated laws of motion applied to all observable movements of inanimate Nature : they governed the motion of waves and projectiles, visible solids and invisible air, resisted as well as free movements. Together with the inverse square law they explained not only the gross movement of planets and the movement of the comets, which before had seemed capricious, but also details which nobody had ever considered as being mechanically explicable, such as the precession of the equinoxes. The problems of the tides and of the irregularities of the moon's motion he did not fully solve, it is true, but he did enough to convince mathematicians that they were soluble by his methods. After Newton's work had been assimilated, the body of natural philosophers accepted it as a commonplace that all terrestrial and celestial movements were explicable in precise and numerical terms by calculations based on a few general laws: before Newton most thinkers were ready to invoke ad hoc principles and occult causes, based on human and divine analogies, for any but the simplest terrestrial phenomena, and the few who were in advance of their times were feeling tentatively for solutions which eluded their grasp.

Even in such thoughts on chemistry as he pub. lished Newton was looking for an explanation in terms of attractions, though, strangely enough, in this science he never seems to have applied his own rule and made quantitative experiments. His work on light lies somewhat outside the mechanical scheme, but here again his insistence on the quantitative created a completely new attitude towards colour. It became a subject for measurement and calculation, rather than one for discussion in terms of generalities.

If we are to try to represent Newton's achievements by some modern analogy, to construct some imaginary figure who should be to our times what Newton was to his, we must credit this synthetic representative with, I think, the whole of relativity up to, and somewhat farther than, the stage at present reached-we must suppose our modern Newton to have satisfactorily completed a unitary field theory. In light we must credit him both with having established the existence of spectral regularities and with their explanation in terms of the quantum theory. Possibly, too, we must give him the Rutherford atom model and its theoretical development, a simple astronomy in little to correspond to the solar system. Let us, then, think of one man who, starting in 1900, say, had done the fundamental work of Einstein, Planck, Bohr and Schrödinger, and much of that of Rutherford, Alfred Fowler and Paschen, say, by 1930, and had then become governor of the Bank of England, besides writing two books of Hibbert Lectures and spending much of his time on psychical research, to correspond with Newton's theological and mystical interests. Let such a man represent our modern Newton and think how we should regard him. Only so, I think, can we see Newton as he appeared to his contemporaries at the end of his life.

There are no discontinuities in Nature and there are none in the history of science. No discovery or fundamental innovation is absolutely new, unconnected with past thought and the stirring spirit of its own time. Newton was not uninfluenced by certain of his immediate predecessors and of his contemporaries. The revolt from the introspective method of constructing explanations of heavenly and earthly phenomena by appeals to philosophic necessity had begun before his birth, and his time was rich in brilliant exponents of the experimenta] philosophy, whose names will always stand as stars adorning the story of science. To compare him with other men of his time and to recognize their contributions to the development of the physical sciences does not, however, lead us to think less of Newton's achievements but rather to wonder at them all the more. It is easier to estimate the size of a Colossus if there are statues of more than life size in its neighbourhood than if it stand alone in a desert.

Newton owed much to the pioneer labours of Galileo, who had founded the science of mechanics, and, in a different way, much to Barrow, whose great mathematical acuteness and sympathetic support were always at the disposal of the young Cambridge scholar. To Hooke he owed more than he was ever prepared to acknowledge. From his great Continental contemporaries, Huygens and Leibniz, he borrowed little, if anything. Possibly if mathematical advances were alone in question Leibniz would have to be considered for a place on the same level as Newton, but for Newton mathematics were merely a means to a physical end: his mathematical innovations may even be left out of consideration without grave injury to Newton's fame. There is no record of physical experimentation that can compare for mastery and elegance with the "Opticks", no work in exact science that produces the same impression of supreme greatness and power of thought that the "Principia" does.

The spirit of this age is a denigrating one, which, 
in a reaction from the earlier custom of representing great men as free from all blemish and weakness, delights in attempting to show that nobody much exceeds the common level. If an earlier painter might have left out Cromwell's warts, the painter of to-day might represent his face as one huge wart. I have read records of Newton's weaknesses and I know of the adumbrations of his discoveries that can be found in forerunners and contemporaries: I acknowledge that his earlier biographer shut his eyes to any incident, writing or action that might seem to detract from his perfection. Nevertheless, all things considered, I think that the contemporary judgment of his greatness can still stand, and that, if the Marquis de l'Hôpital's query as to whether Newton ate, drank or slept like ordinary men ("for I picture him to myself as a celestial genius") seems to our present-day sobriety an affectation, nevertheless we may agree that the line of Lucretius placed on the Trinity statue was well chosen and fitting-

Qui genus humanum ingenio superavit,

"who excelled the human race in power of thought".

\section{NEWTON AS AN EXPERIMENTER \\ BY THE RIGHT HON. LORD RAYLEIGH, F.R.S.}

$\mathrm{T}$ THE duty has been assigned to me of telling you something about Newton as an experimentalist. As the result of a study of what is known of his history, it seems to me that among his various intellectual pursuits experiment was his first love and the love to which he was most constant. Strange though it be, he seems in some moods to have doubted whether his theoretical studies were worth while, and I do not recall any case where he expressed himself enthusiastically about them. On the other hand, he speaks of his optical work as "The oddest, if not the most considerable detection which hath hitherto been made in the operation of nature".

Newton loved the mechanical side of experimental work. As a boy he constructed sundials, and, what is more, fixed one of them into the side of the house effectually enough for it to be there a century later. A notebook of his boyhood shows him assiduous in collecting recipes for various kinds of drawing materials, and he notes methods of performing some (rather nasty) conjuring tricks. Later on, when he is making his reflecting telescope, it is obvious that he is a skilled amateur mechanic, at home in furnace operation. He builds his own brick furnace, prepares speculum metal, and is apparently more successful than the professional opticians of the time in grinding and polishing it to a satisfactory spherical figure. (The days of parabolizing were not yet.) It was not until a good many years later that they were able to put such instruments on the market.*

Asked in his old age where he got the tools for his work, he replied that he had made them himself, and could have achieved little progress without doing so.

There are occasional hints to be gleaned that Newton practised other mechanical arts. Thus, when he examines the colour of thin blown glass, it appears that he has the facilities for glass-blowing at hand, and was presumably able. to use them.

So much for the base mechanical side. Newton, however, had what may be called the itch of experjment and instinctively examined in this way any natural phenomenon that excited his interest. This instinct is not a common one, and it would be of interest to investigate statistically whether it is more correlated with mathematical aptitude than with, say, an aptitude for literary and historical studies. Nowton had all these. Although he experimented in

* Among the first successful commercial makers of reflecting telescopes was James Short (1710-1768), whose instruments were of the Gregorian type. He is said to have made a considerable number of concave and convex mirrors, and to have 'married' them by trial of what pairs gave the best result. other fields, such as mechanics, heat and electricity, and even in anatomy and physiology, his optical experiments are of much greater importance, and in the short time at our disposal we shall only be able to consider a part even of these. The fundamental researches on the composition of white light were read before the Royal Society in 1672, and afterwards recapitulated in his "Opticks" (1704), and we cannot do better than concentrate our attention on them. Although the results are common property nowadays, yet on an occasion like this we shall do well to go back to Newton's own methods and point of view, and to repeat his experiments as nearly as we can in his own way. We must be content to use the positive crater of the electric arc to represent the sun.

Newton's experiments on the spectrum are sometimes presented as if he had started out with the idea of examining the composition of white light. It is true that his "Opticks" (like his "Principia") introduces the various topics as theorems or problems proposed after the manner of Euclid. It does not seem likely, however, that he set out in the first instance to prove any proposition. He bought a prism at Stourbridge Fair (near Cambridge) in 1666, "to try therewith the celebrated phenomena of colours". It is clear from this that the prismatic colours were quite a well-recognized phenomenon at this time, and this is also shown by the circumstance that the art of cutting diamonds so as to display them was already long known. In Peacham's "Gentlemanly Exercises" (1612) reference is made to "A three square cristal prisme wherein you shall perceive the blew to be outmost next to the red", and Grimaldi and others had already experimented on the subject, though without arriving at clear views. We can readily imagine how Newton, handling the prism, would soon find that the colours were well seen in candle-light, but not in diffused daylight. It would not be a long step from this to try the effect on a beam of direct sunlight admitted through a hole in the shutter.

There is no reason to think that he did this with a very elear anticipation of what the effect would be. He was exploring a nearly virgin territory. His beam of sunlight passing through the hole produced an image of the sun. It was what we now call a pinhole image, though the hole need not be very small. Newton's hole was $\frac{1}{8}$ in. diameter. Then he interposed the prism (Fig. 1).

To most people the colours would seem the main 